\title{
A Case of Drug-Induced Interstitial Pneumonia Potentially Related to Quetiapine (Seroquel) Therapy for Behavioral and Psychological Symptoms
}

\author{
Se-Jin Kim MD, Sang-Don Han MD, Jung Yeon Lee MD, and Gyu Rak Chon MD
}

\begin{abstract}
Quetiapine is regarded as an effective and safe treatment for delirium. An 82-year-old man presented with a 1-week history of violent behavior and dizziness accompanied by weakness on the left side of his body. He was diagnosed with acute cerebral cortical infarction and delirium associated with alcohol abuse. After quetiapine treatment, he complained of fever and coughed up sputum, whereas his aggressive behavior improved. His symptoms persisted despite empirical antibiotic treatment. All diagnostic tests for infectious causes were negative. High-resolution computed tomography revealed bilateral consolidations and ground-glass opacities with predominantly peribronchial and subpleural distributions. The primary differential diagnosis was drug-associated interstitial lung disease, and therefore, we discontinued quetiapine and began methylprednisolone treatment. His symptoms and radiologic findings significantly improved after receiving steroid therapy. We propose that clinicians need to be aware of the possibility that quetiapine is associated with lung injury. Key words: quetiapine; alcohol abuse; interstitial pneumonia. [Respir Care 2014;59(10):e145-e148. (C) 2014 Daedalus Enterprises]
\end{abstract}

\section{Introduction}

Quetiapine (Seroquel, AstraZeneca Pharmaceuticals, Wilmington, Delaware) is an atypical antipsychotic medication effective for treating schizophrenia, bipolar disorder, and major depressive disorder. Quetiapine is indicated and approved for the treatment of psychotic disorders in adults by the United States FDA. In addition, quetiapine is regarded as an effective and safe treatment for the symptoms of delirium. ${ }^{1-3}$

Dr Kim is affiliated with the Division of Pulmonary Medicine, Department of Internal Medicine, The Armed Forced Capital Hospital, Bundang-gu, Seongnamsi, Korea. Dr Han is affiliated with the Department of Neurology, Chungju Hospital, Konkuk University School of Medicine, Chungju, Korea. Drs Lee and Chon are affiliated with the Division of Pulmonary and Critical Care Medicine, Department of Internal Medicine, Chungju Hospital, Konkuk University School of Medicine, Chungju, Korea.

The authors have disclosed no conflicts of interest.

Correspondence: Gyu Rak Chon MD, Division of Pulmonary and Critical Care Medicine, Department of Internal Medicine, Chungju Hospital, Konkuk University School of Medicine, 82 Gukwon-daero, Chungju 380-704, Republic of Korea. E-mail: medicor@kku.ac.kr.

DOI: $10.4187 /$ respcare.02977
Quetiapine has several side effects, including adverse effects on the cardiovascular, endocrine, and metabolic systems, such as hypotension, tachycardia, somnolence, prolongation of QT interval, hypothyroidism, and hepatic dysfunction. ${ }^{4-7}$ However, side effects involving the respiratory system are few. A recently published report shows that quetiapine is associated with increased risk of pneumonia at the beginning of treatment, although there is no clear dose-dependent relationship. ${ }^{8}$ In addition, interstitial lung disease potentially related to quetiapine has not been reported. Herein, we describe a patient diagnosed with drug-induced interstitial pneumonia potentially related to quetiapine therapy for behavioral and psychological symptoms.

\section{Case Report}

An 82-y-old man presented with a 1-week history of violent behavior and dizziness accompanied by weakness on the left side of his body. He was a chronic alcohol abuser and had consumed 2 bottles of distilled rice liquor (soju, alcohol content between 10 and 25\%) $5 \mathrm{~d} /$ week for the last 30 years. He had no other significant past medical history and had never smoked.

After admission, the patient received a neurological examination, which revealed an abnormal tandem gait test, 


\section{Drug-Induced Interstitial Pneumonia Potentially Related to Quetiapine}

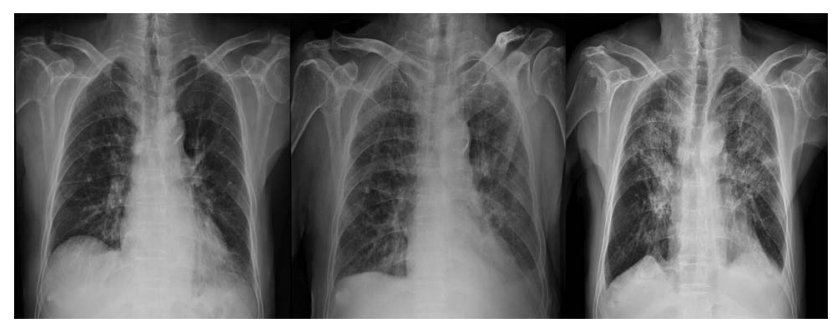

Fig. 1. Left: Chest radiography showed no active parenchymal lesion on admission. Center: Day 5 after initiation of quetiapine, chest radiography showed bilateral patchy and linear opacities in both lungs. Right: After 7 days of antibiotic therapy, chest radiography showed an increase in diffuse patchy opacities in both lungs.

suggestive of abnormal cerebellar function. Subsequent brain magnetic resonance imaging revealed an acute right cerebral cortical infarction. His behavior indicated alcoholic delirium. Consequently, he was admitted to the Department of Neurology at Chungju Hospital in Chungju, Korea, for treatment of cerebral cortical infarction and behavioral symptoms.

The patient's laboratory tests were as follow: hemoglobin, $12.3 \mathrm{~g} / \mathrm{dL}$; white blood cell count, 6,100 cells $/ \mu \mathrm{L}$ (neutrophils, $82.7 \%$; lymphocytes, $7.3 \%$; monocytes, $8.8 \%$; eosinophils, $1.0 \%$; and basophils, $0.2 \%$ ); and platelet count, 129,000 cells $/ \mu \mathrm{L}$. The patient's serum biochemistry tests were as follow: aspartate aminotransferase, $46 \mathrm{IU} / \mathrm{L}$; alanine aminotransferase, $26 \mathrm{IU} / \mathrm{L}$; total bilirubin, $1.2 \mathrm{mg} / \mathrm{dL}$; alkaline phosphatase, $73 \mathrm{IU} / \mathrm{L}$; total protein, $5.5 \mathrm{~g} / \mathrm{dL}$; albumin, $3.0 \mathrm{~g} / \mathrm{dL}$; blood urea nitrogen, $17 \mathrm{mg} / \mathrm{dL}$; creatinine, $1.0 \mathrm{mg} / \mathrm{dL}$; total cholesterol, $108 \mathrm{mg} / \mathrm{dL}$; triglyceride, $398 \mathrm{mg} / \mathrm{dL}$; high-density lipoprotein cholesterol, $31 \mathrm{mg} / \mathrm{dL}$; low-density lipoprotein cholesterol, $65 \mathrm{mg} / \mathrm{dL}$; lactate dehydrogenase, $211 \mathrm{IU} / \mathrm{L}$; and C-reactive protein, $17.90 \mathrm{mg} / \mathrm{dL}$. Chest radiography showed no active parenchymal lesion (Fig. 1, left).

To treat the cerebral infarction, the patient was administered anticoagulant therapy along with platelet aggregation inhibitor (ozagrel sodium, $80 \mathrm{mg}$ twice daily; clopidogrel, $75 \mathrm{mg} / \mathrm{d})$, intravenous naloxone $(10 \mathrm{mg} / \mathrm{d})$, and intravenous mannitol (50 g every $6 \mathrm{~h}$ ). He was also administered intravenous thiamine $(100 \mathrm{mg} / \mathrm{d})$ for prophylactic therapy of alcohol withdrawal syndrome and rebamipide (100 mg 3 times daily) for gastric mucosa protection. On the 2nd day of hospitalization, his behavior became more aggressive; therefore, quetiapine treatment $(50 \mathrm{mg} / \mathrm{d})$ was started. On the 4th day of hospitalization, his neurologic and behavioral symptoms showed improvement. However, he complained of fever and coughed up sputum. His clinical pulmonary infection score was 6 points (body temperature of $38.5^{\circ} \mathrm{C}$, presence of group 4 nonpurulent sputum, local infiltration of chest $\mathrm{x}$-ray, and radiologic progression of pulmonary infiltrates on the left lower lung fields compared to the initial study). Therefore, empirical antibiotic therapy for pneumonia was started (ceftriaxone, $2 \mathrm{~g}$ every $24 \mathrm{~h}$; clindamycin, $600 \mathrm{mg}$ every $8 \mathrm{~h}$ ). On the 5 th day of hospitalization, he experienced a persistent fever; a bronchoscopic examination was performed, and the antibiotic treatment was changed to piperacillin and tazobactam sodium ( 4.5 g every $8 \mathrm{~h}$ ).

Chest radiography showed bilateral infiltrates in both lung fields along with left side pleural effusion (Fig. 1, center). His symptoms persisted into the 7 th day of hospitalization despite 2 days of antibiotic therapy. The patient's vital signs were as follows: blood pressure, 150/90 $\mathrm{mm} \mathrm{Hg}$; pulse rate, 88 beats/min; breathing frequency, 22 breaths/min; and body temperature, $38.3^{\circ} \mathrm{C}$. Laboratory tests showed the following findings: hemoglobin, $11.5 \mathrm{~g} / \mathrm{dL}$; white blood cell count, 9,400 cells $/ \mu \mathrm{L}$ (neutrophils, $84.6 \%$; lymphocytes, $6.1 \%$; monocytes, $5.8 \%$; eosinophils, $3.1 \%$; and basophils, $0.4 \%$ ); and platelet count, 257,000 cells $/ \mu \mathrm{L}$. The patient's serum biochemistry tests were as follow: aspartate aminotransferase, $82 \mathrm{IU} / \mathrm{L}$; alanine aminotransferase, $55 \mathrm{IU} / \mathrm{L}$; total bilirubin, $1.1 \mathrm{mg} / \mathrm{dL}$; alkaline phosphatase, $87 \mathrm{IU} / \mathrm{L}$; total protein, $5.0 \mathrm{~g} / \mathrm{dL}$; albumin, $2.0 \mathrm{~g} / \mathrm{dL}$; blood urea nitrogen, $8 \mathrm{mg} / \mathrm{dL}$; creatinine, $0.7 \mathrm{mg} / \mathrm{dL}$; C-reactive protein, $15.32 \mathrm{mg} / \mathrm{dL}$; and procalcitonin, $0.05 \mathrm{ng} / \mathrm{mL}$.

Follow-up chest radiography showed increased diffuse patchy and linear opacities as well as pleural effusion in both lungs (Fig. 1, right). A high-resolution computed tomography scan of the chest showed bilateral pulmonary consolidations and ground-glass opacities with a predominantly peribronchial and subpleural distribution (Fig. 2). The Legionella and pneumococcal urinary antigen tests were all negative. A rapid influenza antigen test was also negative, but polymerase chain reactions for 16 respiratory viruses were not performed because of his financial limitation. The bronchoscopic washing specimens were all negative for Mycobacterium tuberculosis, which was determined by an acid-fast bacilli smear, polymerase chain reactions, and microbial culture.

Based on the clinical symptoms and the low procalcitonin level, drug-induced interstitial lung disease (DILD)

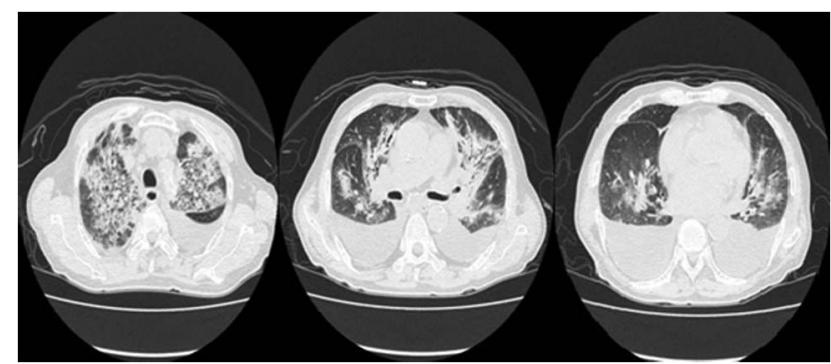

Fig. 2. High-resolution computed tomography scan of the chest showed bilateral pulmonary consolidations and ground-glass opacities with a predominantly peribronchial and subpleural distribution. 


\section{Drug-Induced Interstitial Pneumonia Potentially Related to Quetiapine}

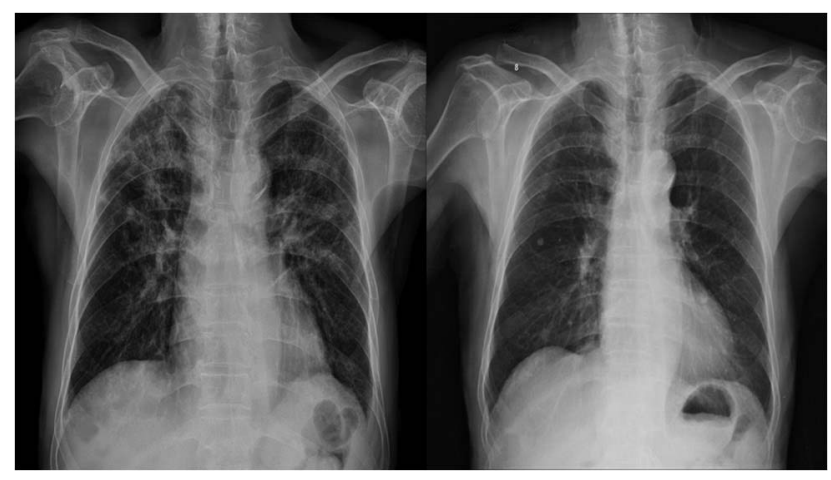

Fig. 3. Left: After discontinuation of quetiapine therapy and 14 days of steroid therapy, chest radiography showed a decreased extent of patch consolidation in both lung fields. Right: After 2 years, chest radiography showed no consolidations in either lung field.

was suspected, and quetiapine treatment and all antibiotics were discontinued. Treatment with methylprednisolone at $50 \mathrm{mg}(1 \mathrm{mg} / \mathrm{kg}$ of body weight) was initiated, and clopidogrel treatment was maintained. After 2 weeks of steroid therapy, his symptoms and radiologic findings significantly improved (Fig. 3, left). The methylprednisolone dosage was gradually decreased to $10 \mathrm{mg} / \mathrm{d}$ for 1 month. The patient did not continue the recommended follow-up visits. However, 2 years later, the patient returned to our hospital and complained of knee pain, and chest radiography showed complete remission with no signs of pleural effusion or bilateral infiltrates noted (Fig. 3, right).

\section{Discussion}

Quetiapine is an atypical antipsychotic medication effective for treating schizophrenia, bipolar disorder, and major depressive disorder. A small, randomized, placebocontrolled trial found significant improvement in noncognitive aspects of delirium with quetiapine. ${ }^{9}$ Although haloperidol is the accepted standard for the treatment of delirious behavior, a study found that treatment with a low dose of quetiapine and haloperidol may be equally effective and safe for treating delirium. ${ }^{10}$

Diagnosis of DILD generally depends upon a definite temporal association between a history of drug therapy and the onset and progression of respiratory complaints. Most importantly, to ensure accurate diagnosis, other causes of lung damage, such as infectious disease, must be excluded. ${ }^{11,12}$ Laboratory findings and clinical manifestations of DILD, such as cough, fever, dyspnea, and hypoxemia, are nonspecific. There are 2 mechanisms involved in DILD that are probably interdependent: one is cytotoxic pulmonary injury, and the other is immune-mediated. Cytotoxic pulmonary injury may occur via reactive oxygen species, reduction in deactivation of metabolites of the lung, impairment of alveolar repair mechanisms, and release of various cytokines. These include cytotoxic drugs, such as bleomycin, methotrexate, and cyclophosphamide, and noncytotoxic drugs, such as nitrofurantoin, sulfasalazine, and amiodarone. Immune-mediated DILD may be mostly $\mathrm{T}$ cell-mediated. For example, in minocycline- and amiodarone-induced interstitial pneumonia, some proportion may result from an immune-mediated mechanism. ${ }^{12}$ In addition, Wijnen et al ${ }^{13}$ indicated that various cytochrome $\mathrm{P} 450$ (CYP) genotypes presented substantial host susceptibility risk for the development of DILD. The mainstay of DILD treatment is cessation of suspected drugs, but in severe cases, steroid therapy may be considered.

A pathological examination was not performed on our patient. However, infectious pathogens were excluded as the cause of interstitial pneumonia, as a microbial culture was negative for infectious bacteria. In addition, the patient's symptoms and infiltrations on chest radiography occurred immediately after initiating quetiapine treatment and quickly improved after discontinuing quetiapine and initiating steroid therapy. Therefore, the patient was presumptively diagnosed with interstitial lung disease potentially related to quetiapine administration.

Several possible mechanisms may explain the occurrence of interstitial lung disease in our patient. First, our patient's chronic history of alcohol abuse may have caused glutathione depletion and increased reactive oxygen species production, which induces oxidative and endoplasmic reticulum stress and can expose the alveolar epithelium and respiratory tract to toxins. ${ }^{14}$ Second, the lungs contain CYP2D6, which is thought to be a minor contributor to CYP-mediated metabolism of quetiapine. ${ }^{15}$ It is considered a high-affinity/low-capacity enzyme, meaning that CYP2D6 will preferentially metabolize drugs at lower concentrations. ${ }^{13}$ Serial liver function tests indicated mild hepatic dysfunction and increased cellular damage. This raises the possibility that CYP3A4 activity was decreased by hepatic dysfunction, and CYP2D6 had a prominent role in metabolizing quetiapine, especially at a lower concentration. In our case, increased quetiapine metabolism by CYP2D6 in the lungs may have increased 7-hydroxyquetiapine, an active metabolite that may damage vital pulmonary tissue. Finally, there are variations in the CYP2D6 alleles across ethnicities. Bradford ${ }^{16}$ found reduced function or nonfunctional CYP2D6 alleles more frequently in Asians, Pacific Islanders, Africans, and African Americans $(40-50 \%)$ than in whites (26\%). This genetic susceptibility may play a role in our patient. Consequentially, we propose that clinicians need to be aware of the potential for quetiapine-induced lung injury.

\section{REFERENCES}

1. Devlin JW, Roberts RJ, Fong JJ, Skrobik Y, Riker RR, Hill NS, et al. Efficacy and safety of quetiapine in critically ill patients with 


\section{Drug-Induced Interstitial Pneumonia Potentially Related to Quetiapine}

delirium: a prospective, multicenter, randomized, double-blind, placebo-controlled pilot study. Crit Care Med 2010;38(2):419-427.

2. Sasaki Y, Matsuyama T, Inoue S, Sunami T, Inoue T, Denda K, Tsukaka K, Koyama T. A prospective, open-label, flexible-dose study of quetiapine in the treatment of delirium. J Clin Psychiatry 2003; 64(11):1316-1321.

3. Schwartz TL, Masand PS. Treatment of delirium with quetiapine. Prim Care Companion J Clin Psychiatry 2000;2(1):10-12.

4. Balit CR, Isbister GK, Hackett LP, Whyte IM. Quetiapine poisoning: a case series. Ann Emerg Med 2003;42(6):751-758.

5. Eyer F, Pfab R, Felgenhauer N, Strubel T, Saugel B, Zilker T. Clinical and analytical features of severe suicidal quetiapine overdoses-a retrospective cohort study. Clin Toxicol 2011;49(9):846-853.

6. Feret BM, Caley CF. Possible hypothyroidism associated with quetiapine. Ann Pharmacother 2000;34(4):483-486.

7. Shpaner A, Li W, Ankoma-Sey V, Botero RC. Drug-induced liver injury: hepatotoxicity of quetiapine revisited. Eur J Gastroenterol Hepatol 2008;20(11):1106-1109.

8. Kuo CJ, Yang SY, Liao YT, Chen WJ, Lee WC, Shau WY, et al. Second-generation antipsychotic medications and risk of pneumonia in schizophrenia. Schizophr Bull 2013;39(3):648-657.

9. Tahir TA, Eeles E, Karapareddy V, Muthuvelu P, Chapple S, Phillips $\mathrm{B}$, et al. A randomized controlled trial of quetiapine versus place- bo in the treatment of delirium. J Psychosom Res 2010;69(5):485490.

10. Maneeton B, Maneeton N, Srisurapanont M, Chittawatanarat K. Quetiapine versus haloperidol in the treatment of delirium: a doubleblind, randomized, controlled trial. Drug Des Devel Ther 2013;7: 657-667.

11. Shi JH, Yan XW, Xu WB, Liu HR, Zhu YJ. [Clinicopathological manifestations of drug-induced lung injury]. Zhonghua Jie $\mathrm{He} \mathrm{He} \mathrm{Hu}$ Xi Za Zhi 2007;30(3):161-166. Article in Chinese.

12. Matsuno O. Drug-induced interstitial lung disease: mechanisms and best diagnostic approaches. Respir Res 2012;13:39.

13. Wijnen PA, Bekers O, Drent M. Relationship between drug-induced interstitial lung diseases and cytochrome $\mathrm{P} 450$ polymorphisms. Curr Opin Pulm Med 2010;16(5):496-502.

14. Kaphalia L, Calhoun WJ. Alcoholic lung injury: metabolic, biochemical and immunological aspects. Toxicol Lett 2013;222(2):171179 .

15. DeVane CL, Nemeroff CB. Clinical pharmacokinetics of quetiapine: an atypical antipsychotic. Clin Pharmacokinet 2001;40(7):509-522.

16. Bradford LD. CYP2D6 allele frequency in European Caucasians, Asians, Africans and their descendants. Pharmacogenomics 2002; 3(2):229-243. 